\title{
Management of blunt hepatic and splenic trauma in Austria: a national questionnaire study
}

\author{
Margot Fodor · Florian Primavesi - Dagmar Morell-Hofert - Matthias Haselbacher - Eva Braunwarth · \\ Benno Cardini · Eva Gassner · Dietmar Öfner · Stefan Stättner (D)
}

Received: 18 December 2018 / Accepted: 18 March 2019 / Published online: 3 April 2019

(C) The Author(s) 2019

\begin{abstract}
Summary
Background Treatment of hepatic and splenic injuries has significantly evolved over the past 30 years: Nonoperative management (NOM) has increasingly become standard of care for the majority of patients in specialised centres. However, patient selection and details of practical management such as time to reinitiating oral intake, duration of restricted activity, or necessity of repeated imaging are still a matter of debate. This national multicentre questionnaire study aims to give a cross-sectional overview of current management of blunt liver and splenic trauma in Austrian hospitals.
\end{abstract}

\footnotetext{
Open Access This article is distributed under the terms of the Creative Commons Attribution 4.0 International License (http://creativecommons.org/licenses/by/4.0/), which permits unrestricted use, distribution, and reproduction in any medium, provided you give appropriate credit to the original author(s) and the source, provide a link to the Creative Commons license, and indicate if changes were made.
}

Electronic supplementary material The online version of this article (https://doi.org/10.1007/s10353-019-0586-9) contains supplementary material, which is available to authorized users.

M. Fodor · F. Primavesi · E. Braunwarth · B. Cardini .

D. Öfner · S. Stättner, MD ( $\varangle)$

Department of Visceral, Transplant and Thoracic

Surgery, Medical University of Innsbruck,

Anichstraße 35, 6020 Innsbruck, Austria

stefan.staettner@i-med.ac.at

D. Morell-Hofert · E. Gassner

Department of Radiology, Medical University of Innsbruck, Anichstraße 35, 6020 Innsbruck, Austria

\section{Haselbacher}

Department of Trauma Surgery, Medical University of

Innsbruck, Anichstraße 35, 6020 Innsbruck, Austria
Methods The survey was addressed to all Austrian surgical departments and trauma units. After three months, responses were electronically and anonymously recorded, data were analysed using descriptive statistics. Data collection involved electronicbased questionnaires comprising questions on centre structure, selection criteria for NOM and practical aspects of consecutive treatment.

Results In total, a $60 \%$ response rate could be achieved, and $24 \%$ of all contacted centres filled out the full questionnaire completely. A widespread shift to NOM within recent years could be observed. More than $70 \%$ of injuries were treated conservatively. Forty percent of hospitals currently follow a clinical algorithm. Further details about specific questionnaire results are presented, revealing diverse approaches in a number of treatment aspects.

Conclusion Non-operative management is the standard of care for blunt hepatic and splenic injuries in Austria. In many clinically relevant questions there is still a lack of consensus. Based on this experience, national standard protocols may be generated for systematisation of care in blunt liver and spleen trauma.

Keywords Liver injury · Splenic injury · Non-operative management $\cdot$ Survey $\cdot$ Liver and spleen

\section{Background}

Blunt abdominal injuries, frequently due to sports and traffic accidents, represent a challenging and common cause of trauma in central Europe. Management of these injuries can be difficult because of the frequent association with a multifaceted clinical picture of additional thoracic, limb and head injuries [1, 2]. Over the past few years, non-operative management (NOM) of blunt splenic and liver injuries has become the standard of care in haemodynamically stable pa- 
tients [3, 4]. In general, abdominal organs are involved in about one third of polytrauma patients, with an incidence of hepatic and splenic injuries of 13 and $16 \%$, respectively $[2,4]$.

Therapeutic approaches comprise either surgical treatment or NOM, which as commonly defined also include nonsurgical measures such as endoscopic (e.g. bile duct stenting) or radiological interventions (angiography, coiling, etc.). Due to frequent postoperative complications after primary surgical treatment, in recent decades a paradigm shift to NOM has been established in major trauma centres [4]. This change was propelled by intraoperative observations that found many minor liver and splenic injuries no longer bleeding [5, 6], with no need for further surgical interventions. Besides avoidance of unnecessary, non-therapeutic laparotomies, frequent application of NOM resulted in additional benefits such as decreased use for blood transfusions, lower mortality rates and lower healthcare costs [7, 8].

To employ a NOM pathway for cases with blunt hepatic and splenic injuries requires the patient to meet several criteria, primarily haemodynamic stability. Although many institutional protocols addressing NOM include contraindications such as advanced patient age, higher grade injury or a certain number of initially applied transfusions, these factors represent individual recommendations mostly not based on high-level scientific evidence. The recent 2012 edition of the Eastern Association for the Surgery of Trauma (EAST) guidelines for the management of blunt hepatic injuries are similar to those for blunt splenic injuries with possibility of extra interventions including angiography, endoscopic retrograde cholangio-pancreaticography (ERCP) and laparoscopy [8]. Haemodynamically unstable patients or those affected by peritonitis still necessitate operative intervention. However, many questions regarding practical aspects of NOM remain without conclusive answers within the guidelines, despite the high number of recent publications on this topic [9]. For example, there are few clear recommendations regarding several clinically important aspects: frequency of clinical examination, imaging procedures and haemoglobin measurements; intensity and duration of monitoring, levels of transfusion triggers after which operative or angiographic intervention should be considered, time to re-initiating oral intake, duration and intensity of restricted intra-hospital and post-discharge activity, optimum length of stay (LOS) within the ICU and hospital, timing of initiating venous thromboembolism prophylaxis and postdischarge follow-up [7].

In Austria, according to national data [10], the most frequent causes of blunt abdominal injuries are traffic and sport accidents. In $2017,15,000$ to 35,000 sport accidents per year and 60 to 100 car accidents per day were registered. In Austria, abdominal trauma management is mostly performed by general surgeons. As a consequence of existence of only a few high-vol- ume centres, most injuries are primarily treated in level II-III hospitals, where expertise and resources are not comparable with those of acute care general hospitals (level I). In the absence of defined nationwide protocols, each single centre treats patients individually. This multicentre questionnaire study was designed to give a cross-sectional overview of current management of blunt liver and splenic trauma in Austrian hospitals. The survey includes specific questions on basic treatment strategies and practical aspects of NOM aiming to address the following main areas of controversy: (1) treatment algorithms for blunt abdominal injuries in individual centres, (2) volume of treated patients, rates and criteria for NOM and (3) mobilisation, thromboprophylaxis, follow-up and return-to activity after trauma.

Based on the survey outcome, unclear aspects of NOM in clinical practice may be standardised nationally.

\section{Methods}

\section{Study design}

A national survey including 24 questions to collect information on department structure and opinions about areas of controversy in NOM of hepatic and splenic injuries was generated. The following steps were conducted for upfront validation of the questionnaire: translation of the questionnaire into German, expert content evaluation (interview with experienced trauma surgeons at our high-volume centre in Innsbruck), test on reliability, comparison to other surveys.

\section{Selection of participants and questionnaire design}

Chief surgeons of all Austrian departments involved in general and trauma surgery (regardless of unit size) were selected to represent a broad range of expertise. In all, 102 surgical units and 103 trauma units participated in three repeated rounds of the survey, to ensure an adequate response rate. Each centre was initially contacted through an invitation letter and email, including an information sheet and the firstround survey. Surveys were to be completed within two weeks, a reminder was sent to those experts who had not submitted responses within this time period. In summary the survey was sent out three times between October and December 2017 using a mailing list. Specifically, experts received a questionnaire with 24 questions addressing the specific areas of clinical controversy to assess the range of diversity in clinical practice. The survey was designed utilizing Survio (Survio s.r.o., Czech Republic, http:// www.survio.com/en/online-surveys-version 2017) and was originally written in the German language. An English version was created for this publication (Supplement A1). 


\section{Statistical analysis}

Descriptive statistics were performed to report frequencies of survey results. Differences between highand low-volume centres were analysed with the Pearson chi-square test or the Fisher's exact test. $P$-values $<0.05$ were considered to be statistically significant. Data analysis was carried out using SPSS version 21 (IBM Inc., Armonk, New York, USA).

\section{Results}

\section{Response rates}

In summary, 205 trauma surgery and general surgical units were contacted, of which $61 \%$ responded to the survey $(n=125)$, either partially $(64 \%, n=80)$ or completely (38\%, $n=48)$. All but three institutions confirmed, they were regularly treating blunt hepatic and splenic injuries recently. To facilitate correct statistical analysis, only fully completed surveys (36\%, $n=45)$ were considered for final evaluation.

\section{Overview institution data}

Institutions were differentiated into Acute Care General Hospitals (level I; 47\%; $n=21$ ), Specialty Acute Care Hospitals (level II; $42 \% ; n=19$ ) and Teaching/ University Hospitals (level III; $11 \% ; n=5$ ). In $69 \%$ of cases $(n=31)$, departments of general surgery were responsible for blunt abdominal trauma, in $9 \%(n=4)$ departments of trauma surgery and in $13 \%(n=6)$ the decision was made case by case. Interdisciplinary management was performed by $4 \%(n=2)$ of institutions, while $4 \%(n=2)$ affirmed that this depended on grade of concomitant injuries.

When asking about opinions on the appropriate environment for treatment of blunt hepatic/splenic injuries, $40 \% \quad(n=18)$ stated that this is primarily a question of available infrastructures, like angiography or ERCP; $33 \%(n=15)$ expressed the opinion that each surgeon has to be able to handle the manage- ment procedures; $20 \%(n=9)$ stated that maximum care hospitals may be required only for complex cases; $4 \%(n=2)$ that these kind of injuries should always be treated in maximum care hospitals, while one participant $(2 \%)$ replied that each hospital with an emergency room may be allowed to treat blunt abdominal traumata.

\section{Therapeutic data and treatment evolution}

In 2016, most centres were treating 5-20 patients per year (Fig. 1a) and the rate of NOM was $>50 \%$ in more than two thirds of all centres (Fig. 1b). The therapeutic approach to isolated splenic injuries in the last 5 years (2011-2016) moved towards conservative management in $60 \%(n=27)$ and towards operative management (splenectomy) in $4 \%(n=2)$; an increasing trend towards interventional radiology was reported in $7 \%(n=3)$ and no obvious change in treatment in $24 \%(n=11)$.

\section{Definitions and classification systems}

The definition of NOM was substantially differed in individual centres: $67 \%(n=30)$ described NOM as absence of any invasive or interventional treatment but $31 \%(n=14)$ also considered interventions like angiography and ERCP as conservative treatment; one responder defined any approach leading to organ preservation as NOM (for example surgical splenorrhaphy).

Regarding usage of a radiological classification of liver/spleen injuries, 53\% ( $n=24)$ did not routinely use a classification system, while $47 \%(n=21)$ did apply a grading system. The American Association for the Surgery of Trauma (AAST/Moore) score for liver (I-VI) and spleen (I-V) injuries is currently the most used system. Other individual classifications were applied at single institutions such as involvement of capsular trauma or differentiation between primary and secondary rupture. In all, 65\% $(n=29)$ agreed that classifying these injuries is helpful in clinical management,

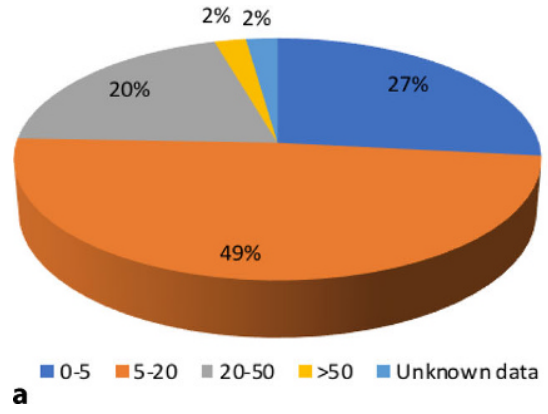

Fig. 1 Survey questions giving an overview of institutional data. a Displaying the number of patients per institution treated with blunt hepatic/splenic injury. Survey question: How many patients with blunt heptic or splenic injuries were

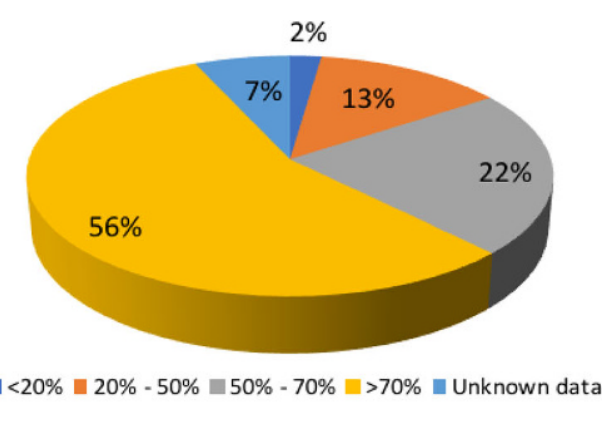

treated at your institution in 2016 ? b Displaying the percentage of patients treated conservatively (with non-operative management). Survey question: How many patients were treated conservatively? 


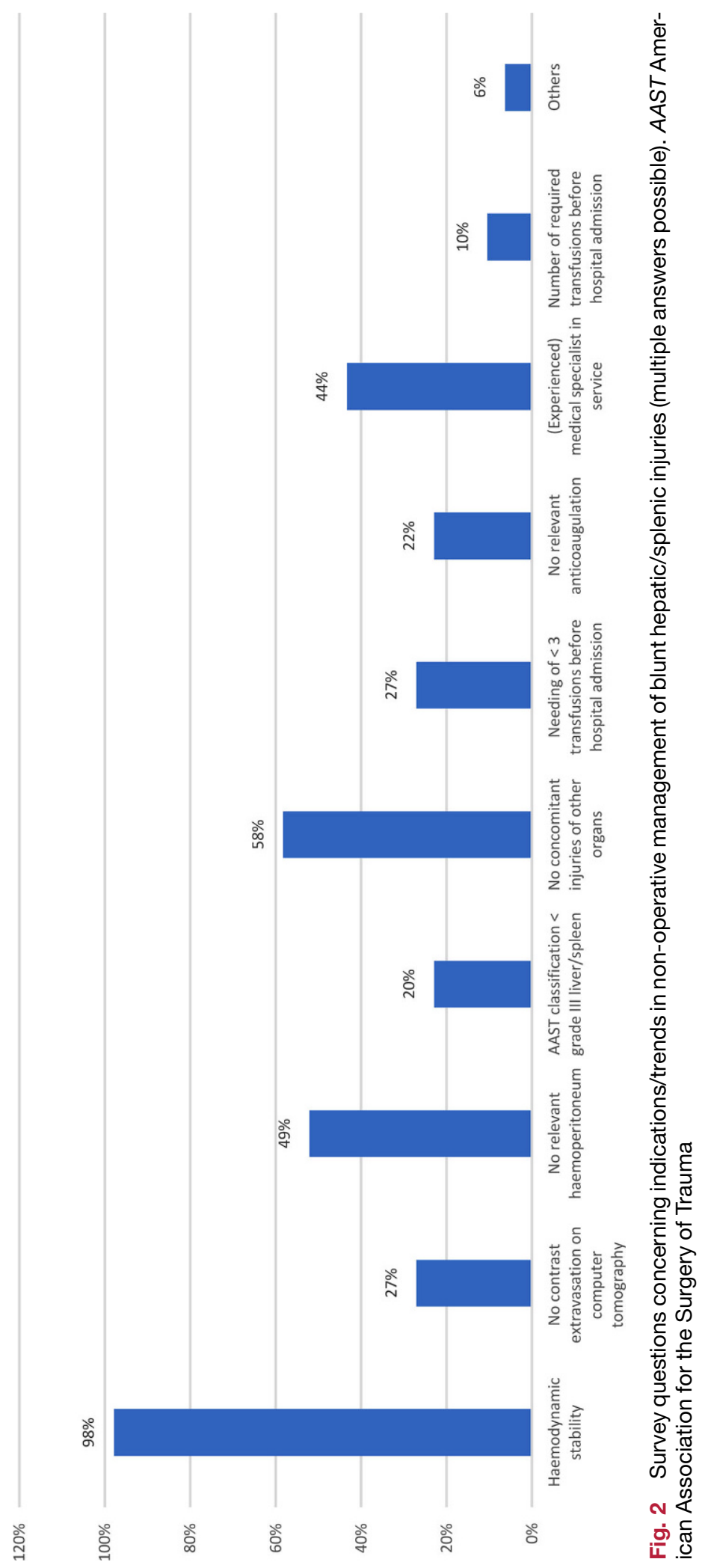


Fig. 3 Survey questions concerning practical aspects in non-operative management of blunt hepatic/splenic injuries. a Timing of post-traumatic mobilisation-When do you allow post-traumatic mobilisation in blunt hepatic/splenic injuries? b Recommended time-point of return to activity -We recommend return to activity after

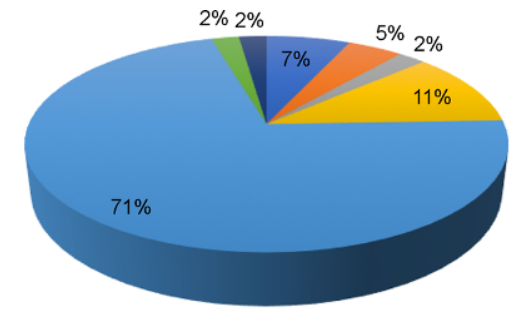

- Mobilisation on first post -traumatic day

- Mobilisation on second post-traumatic day

- Mobilisation on third post-traumatic day

= Mobilisation always after third post-traumatic day

= This is an individual decision

$=$ Partial bed restrain (for example toilet) is always immediately allowed

- Others

a

$28 \%(n=13)$ did not see a value in a classification system, while $7 \%(n=3)$ would use a standardised classification only in individual cases and in combination with clinical parameters.

\section{Indication and practical aspects in NOM of hepatic and splenic injuries}

Definition of criteria needing to be fulfilled for NOM of blunt hepatic/splenic injuries is still a matter of debate in the literature. Austrian institutions (Fig. 2) identified haemodynamic stability $(98 \% ; n=44)$ and no concomitant injuries of other organs $58 \%(n=26)$ as the most relevant criteria; absence of a significant haemoperitoneum was regarded as relevant in $49 \%$ $(n=22)$. Age $>55$ years did not play a significant role.

Timing of post-traumatic mobilisation in blunt hepatic/splenic injuries is based on individual decision in $71 \%(n=33)$ of institutions. Many centres allow mobilisation on standardised time points (Fig. 3a). Routine follow-up imaging after blunt hepatic/splenic trauma was performed very differently according to the answers: $36 \%(n=16)$ did not perform routine follow-up imaging, 26\% ( $n=12)$ performed monthly follow-up imaging with ultrasound, $22 \%(n=10)$ individualised follow-up depending on injury grade,
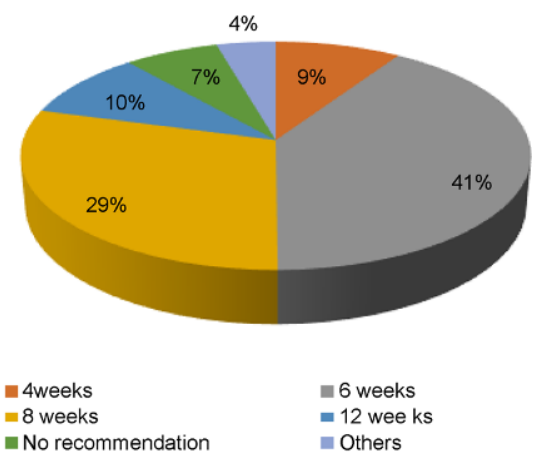

b
$11 \%(n=5)$ initially performed monthly follow-up imaging with ultrasound and a final CT scan after 3-6 months, while $4 \%(n=2)$ of hospitals suggested follow-up imaging with concomitant CT scans after 3-6 months. Return to full daily and moderate sports activity were recommended after at least 6 weeks in two thirds of evaluated centres, but substantially differed between the remaining institutions (Fig. 3b).

Another important clinical aspect in the survey was the optimal timing for initiation of venous thromboembolism prophylaxis. Thirty-six per cent $(n=16)$ of institutions answered this should be an individual decision. Prophylaxis of thromboembolic events with low-molecular weight heparin (LMWH) was started $>48 \mathrm{~h}$ after trauma in $27 \%(n=12)$ of centres, while $9 \%$ ( $n=4)$ of hospitals started LMWH $<48 \mathrm{~h}$. One hospital ( $2 \%)$ started after $24 \mathrm{~h}$, while $22 \%(n=10)$ began immediately after trauma. One centre $(2 \%)$ did not recommend prophylaxis of thromboembolic events, arguing with a higher risk of bleeding in trauma patients. Another institution (2\%) indicated only physical therapy for prophylaxis, avoiding medical anticoagulation.

Concerning potential consequences of splenectomy, $64 \%(n=29)$ of centres recommended spleen preservation with all possible resources as an important goal. Twenty-seven per cent $(n=12)$ answered

Fig. 4 Relevant criteria for demission from intermediate or intensive care units (multiple answers possible)

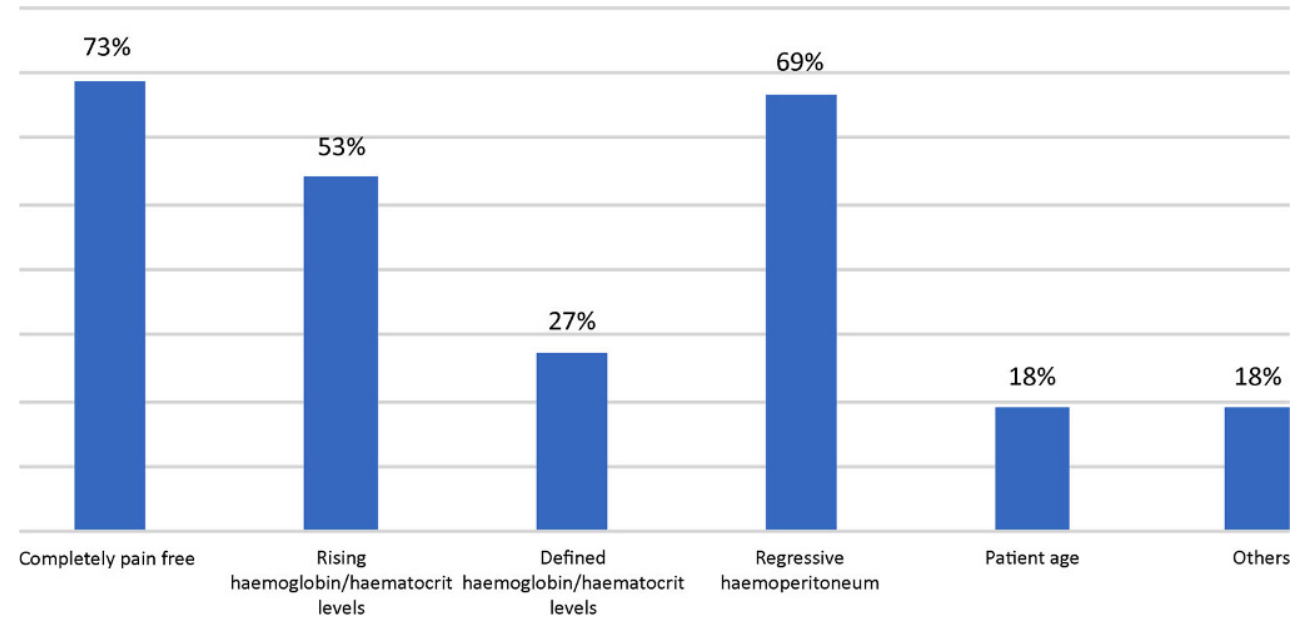


Table 1 Differences in the management between high- (HVC) and low-volume (LVC) centres

\begin{tabular}{|c|c|c|c|}
\hline Variable & $\begin{array}{l}\text { Level II or III hospitals; HVC: } \\
n=24(\%)\end{array}$ & $\begin{array}{l}\text { Level I hospitals; LVC: } \\
n=21(\%)\end{array}$ & $p$-value \\
\hline Primary responsible discipline & & & 0.219 \\
\hline General surgeons & $15(63)$ & $16(76)$ & \\
\hline Trauma surgeons & $2(8)$ & $3(14)$ & \\
\hline Interdisciplinary & $8(29)$ & $2(10)$ & \\
\hline Number of treated blunt abdominal injuries per year & & & $<0.001$ \\
\hline $0-5$ & $1(4)$ & $11(52)$ & \\
\hline $5-20$ & $13(57)$ & $9(43)$ & \\
\hline $20-50$ or more & $9(39)$ & $1(5)$ & \\
\hline Rate of NOM & & & 0.052 \\
\hline$<50 \%$ & $5(23)$ & $2(10)$ & \\
\hline $50-70 \%$ & $2(9)$ & $8(40)$ & \\
\hline$>70 \%$ & $15(68)$ & $10(50)$ & \\
\hline Use of a classification system & & & 0.136 \\
\hline No & $10(42)$ & $14(67)$ & \\
\hline Yes (mostly AAST/Moore) & $14(58)$ & $8(33)$ & \\
\hline Use of a standardised algorithm & & & 1.000 \\
\hline No, every patient treated individually & $13(54)$ & $11(52)$ & \\
\hline Yes, routinely or currently under development & $11(46)$ & $10(48)$ & \\
\hline Timing of mobilisation & & & 0.289 \\
\hline Day 1 & $3(13)$ & 1 (5) & \\
\hline Day 2 & $0(0)$ & $2(9)$ & \\
\hline Day 3 or later & $2(8)$ & $4(19)$ & \\
\hline Individually & $19(79)$ & $14(67)$ & \\
\hline Routine follow-up imaging & & & 0.228 \\
\hline Yes, always (in different intervals) & $11(48)$ & $6(29)$ & \\
\hline No, not routinely & $12(52)$ & $15(71)$ & \\
\hline Start of anticoagulation & & & 0.254 \\
\hline No anticoagulation & $1(4)$ & $1(4)$ & \\
\hline Within the first day & $8(33)$ & $2(10)$ & \\
\hline From day 2 on & $3(13)$ & $2(10)$ & \\
\hline From day 3 on & $4(17)$ & $8(38)$ & \\
\hline Individually & $8(33)$ & $8(38)$ & \\
\hline Audits performed in department & & & 0.501 \\
\hline Never or only special cases & $19(79)$ & $14(67)$ & \\
\hline Yes, regular & $5(21)$ & $7(33)$ & \\
\hline Estimated risk of secondary splenic injury & & & 0.177 \\
\hline $0-10 \%$ & $15(63)$ & $10(48)$ & \\
\hline $10-20 \%$ & $8(33)$ & $6(28)$ & \\
\hline $20-30 \%$ & $1(4)$ & $5(24)$ & \\
\hline Recommended re-initiation of sports activities & & & 0.927 \\
\hline After 4 weeks & $2(8)$ & $2(9)$ & \\
\hline After 6 weeks & $12(50)$ & $8(38)$ & \\
\hline After 8 or 12 weeks & $8(33)$ & $9(43)$ & \\
\hline No recommendation or individually & $2(8)$ & $2(10)$ & \\
\hline Is the management of blunt trauma systemised in Austria? & & & 0.551 \\
\hline No, there is need for guidelines & $22(92)$ & $20(95)$ & \\
\hline Yes, no need for guidelines & $2(8)$ & $1(5)$ & \\
\hline
\end{tabular}


that the indication should be evaluated carefully in older patients, depending on comorbidities. Three hospitals $(7 \%)$ replied that the indication for splenectomy should be legitimated easily due to low risk of long-term consequences. One single institution (2\%) answered potential consequences of splenectomy could be estimated as irrelevant after the age of 20 years. The risk of secondary spleen rupture was estimated $0-10 \%$ in $57 \%(n=25)$ of institutions, $10-20 \%$ in $31 \%(n=14)$ and $20-30 \%$ in $13 \%(n=76)$ of centres. All participating hospitals $(n=45)$ perform vaccination only after splenectomy but not in case of splenic artery embolisation, or purely conservative management. Departments were asked if the potential risk of secondary spleen rupture influenced the time of hospital discharge: $13 \%(n=6)$ answered it could be possible, $69 \%(n=31)$ agreed, $13 \%(n=6)$ responded probably not, $4 \%(n=2)$ denied any influence on discharge dates.

Additionally, relevant criteria for reduction in the intensity of observation were analysed in the questionnaire. Regressive haemoperitoneum was relevant in $69 \%(n=31)$, completely pain free in $73 \%(n=33)$ and rising haemoglobin/haematocrit levels in 53\% ( $n=24)$ among several other criteria for decisionmaking (Fig. 4).

\section{Standardised pathways and algorithms for NOM}

Departments were asked if a standard algorithm in management of hepatic/splenic injuries is routinely applied: $40 \%(n=18)$ adhered to a standard algorithm, while $51 \%(n=23)$ declared that each patient underwent individual treatment. In $7 \%(n=3)$ of responders a standardised procedure was in preparation. One centre $(2 \%)$ reported standardised management and an individualised follow-up. Sixty per cent $(n=27)$ agreed that a standardised algorithm for NOM of blunt hepatic/splenic injuries would be helpful. In contrast, $31 \%(n=14)$ reported that algorithms were not necessary because conservative treatment is always individualised. The remaining 9\% $(n=4)$ mentioned that even if standard procedures are recommended in the literature, individual management should be performed.

Institutions were asked if routine audits of clinical outcomes and failure rate of NOM were in place. Only $27 \%(n=12)$ of centres routinely conduct post trauma outcome audits. Seventy-one per cent $(n=32)$ discussed only individual cases, while one centre (2\%) never audits their results in an interdisciplinary setting. Finally, participants of the survey were asked if they feel that the management of blunt hepatic/ splenic injuries in Austria is actually standardised: 7\% $(n=3)$ agreed, while the majority $(93 \% ; n=42)$ disagreed.

Additionally, differences in the management between high-volume centres (HVC; level II and III) and low-volume centres (LVC; level I) were analysed. As shown in Table 1, only the number of treated blunt abdominal injuries per year $(p<0.001)$ and the rate of NOM ( $p=0.052)$ differed significantly between HVC and LVC. Regarding all other parameters, no pattern of relevant differences could be identified.

\section{Discussion}

Blunt hepatic and splenic injuries are common and NOM shows increasing popularity although good evidence from well-designed prospective trials is lacking [11]. Our study provides a snapshot of the management of these common injuries in Austria. With a response rate of $61 \%$, either partially (64\%) or completely (36\%), the current study achieved a reasonable sample size with an equal distribution of level 1 and higher-level hospitals [11]. For the final evaluation, only completely responses were considered $(n=45)$, representing $22 \%$ of all Austrian surgical and trauma departments (total $n=205$ ). Treatment of patients with extensive injuries has been recommended to be reserved to specialised centres where abdominal surgery can readily be performed in case of failure of NOM. Alternatively, if NOM is failing in a small volume centre, damage control may be performed before referral to a specialised centre for further treatment [11]. The advocated centralisation of specialised procedures at HVC has not been fully performed yet.

In approximately $70 \%$ of responding departments', conservative treatment was implemented in more than $50 \%$ of cases in 2016, comparable to reports in the literature, where NOM was successfully applied in about $60 \%$ of blunt abdominal injuries [4, 12], in Austria experience with comparably large patient series cannot be expected at the current state of our health service structure, where only one institution treated more than 50 patients yearly. According to guidelines, management of liver trauma should be carried out in a specialised centre with a well-trained team, including dedicated liver surgeons, experienced anaesthetists and interventional radiologists with expertise in complex cases and management of complications $[7,8]$. In our survey, only $20 \%$ meant that only maximum care hospitals may be competent to treat complex cases, while $33 \%$ argued that each hospital with an emergency room should be allowed to treat blunt abdominal trauma. A further $40 \%$ of institutions answered that this is primary a question of infrastructure. Other European health care systems are increasingly developing centralised care models as a way to improve outcome of complex procedures. Publications have attested that centralisation can improve clinical outcomes through increased compliance with clinical practice guidelines [11].

As described by further reports [13, 14], a trend towards conservative management in hepatic/splenic injuries could also be detected over the last 5 years (2011-2016) in most institutions in Austria. However, the definition of NOM showed considerable variability 
in Austrian centres. Although the recent 2012 edition of the EAST guidelines explicitly includes angiography, ERCP and laparoscopy in the definition of hepatic/splenic NOM [8], 67\% of Austrian centres still described NOM as absence of any invasive treatment, while only $31 \%$ considered these additive interventions as conservative treatments.

Even if the majority of centres (64\%) expressed the opinion that a common language for specific organ injuries would facilitate clinical decision-making, only $47 \%$, mostly in LVC, used a grading system. The reason could be that in the majority of cases, scoring is only related to the degree of injury in a particular organ, while the outcome depends on the sum of all injuries (especially brain injury) and pre-existing co-morbidities. Correct injury grading depends on high expertise in radiology. Therefore, most centres rely on algorithms combining different parameters such as haemodynamic stability, imaging findings and timely availability of interventional techniques to decide whether to opt for immediate surgery or to attempt NOM. The AAST/Moore score for liver (I-VI) and spleen (I-V) injuries was the most used. This classification scheme is considered the gold standard for organ injury classification [15]. However, some evidence shows that this scoring system alone is not appropriate to direct necessity of operative management or predict outcome of patients with liver or spleen injury $[14,16]$.

In 2016 and 2017, guidelines defining clear indications for NOM in blunt trauma patients were published $[14,16]$. In the present survey institutions selected haemodynamic stability $(98 \%)$, no concomitant injuries of other organs (58\%), no relevant haemoperitoneum $(49 \%)$ and the presence of experienced specialists in service $(43 \%)$ as the most relevant criteria, while patient age did not play a significant role. This is in line with current guideline recommendations.

As expected, in our survey post-traumatic mobilisation in blunt hepatic/splenic injuries was an individual decision in $71 \%$, based on clinical status and vital parameters, independently of the patient volume treated in individual hospitals. Many surgeons still believe that early mobilisation of patients with blunt solid organ injuries increases the risk of delayed haemorrhage. According to London et al. [17], long periods of bed-rest are unnecessary, since the timing of mobilisation does not contribute to late bleeding. In a retrospective cohort study of hepatic injuries, the median time-point of post-trauma mobilisation was day 2; patients with blunt splenic injury were mobilised after median day 3 . There was no significant difference regarding those who failed NOM in the early versus late mobilization groups [17]. On the contrary, there may be significant benefits of early mobilisation such as reduced hospital length of stay (LOS) and reduced resource utilization [17], as well as lower incidence of pneumonia, deep venous thromboembolism (DVT) and pulmonary embolism [18].
To date, high-level evidence based recommendations concerning timing and type of follow up imaging (CT vs. US) are lacking and therefore, individual follow-up is usually based on clinical judgment [14, $16]$. In our study $36 \%$ of centres do not recommend routine follow-up imaging after discharge (especially HVC), while $27 \%$ perform monthly follow-up ultrasounds. The accuracy of abdominal CT in diagnosing liver/spleen trauma excluding other injuries plays a fundamental role in achieving effective NOM [19]. Clancy et al. reported that patients with higher-grade injuries were more likely to receive follow-up imaging during their period of in-hospital stay. Although CT-based documentation of complete organ healing was once standard of care, follow-up CT is no longer recommended unless clinically indicated [20]. Lynch et al. [21] prospectively showed that the average time to sonographic healing in AAST grade I, II, III, and IV injuries was $3,8,12$, and 21 weeks, respectively. Patients returned to full activity with no long-term impediments after ultrasound verified healing [21]. More than half of patients present CT-based restitution after 6 weeks and further intensified follow-up with regular imaging seems to have no clinical value. Complete healing of all grades is shown 3 months after trauma $[14,16]$. Ultrasound follow-up, based on clinical judgment, should be suggested in young patients with low grade injuries $[22,23]$. The main reason for follow-up imaging is delayed splenic rupture. Another motive for bleeding is splenic artery pseudoaneurysms that can even appear in low-grade injuries. Factors predicting this pathology do not exist [22]. Allins et al. published that follow-up scans did not show advance of injury, thus making them superfluous in clinical decision making [24].

Initiation of thromboprophylaxis with LMWH in our country is timed quite individually according to our survey, although crucial in the management of patients after blunt liver or spleen injuries. Especially, the optimal timing for trauma patients with a high risk of bleeding is undefined. EAST supports the practice of early DVT prophylaxis for solid organ injuries but these recommendations lack a clear demarcation of the ideal timing for initiation [25]. A retrospective study showed that early use of LMWH during the first $48 \mathrm{~h}$ of hospitalization was not associated with increased risk for bleeding or failure of NOM [26]. Furthermore, the use of LMWH within the first 48-72h of admission was not combined with increased blood transfusion rate, bleeding complications or NOM failure in studies of other groups $[27,28]$. Hence, recent guidelines recommend early start of mechanical prophylaxis and LMWH-based prophylactic anticoagulation to diminish the rate of thromboembolic complications [14].

Optimal timing of return to full activities also lacks clear evidence in the literature [7, 8]. Most authors associate their recommendation to the gravity of trauma. In the present survey, the majority (41\%) 
recommended return to full daily and moderate sport activity after 6 weeks. Significant differences between HVC and LVC could not be shown. Available literature suggests return to unrestricted activity after splenic trauma is safe at 3 weeks for low grade and at 6 months for severe grade injuries [20]. Another recent survey among experts showed diverging results: nearly half of experts tolerated return to mild activities within 4 to 6 weeks and to full activities within 2 to 3 months in grade III-IV. However, the other half suggested restricted activities for a period of 4-6 months [29]. Some specialists even recommend no unrestricted activity until full organ reconstitution verified on CT, typically 3 to 6 months after injury [20]. Acknowledging the present lack of clear evidence, clinical judgment still represents the key feature in this specific issue.

Adverse outcome in splenic trauma may also occur secondary due to delayed subcapsular hematoma or pseudoaneurysm rupture. Delayed splenic rupture is defined as occurring $48 \mathrm{~h}$ after trauma. Although it is reported that lethal complications after severe abdominal injury mostly occur 1 to $6 \mathrm{~h}$ after onset, there are single reports of secondary rupture occurring up to 70 days later [30]. Delayed rupture of the spleen continues to have a significant mortality rate, despite improvements in imaging and treatment options [30]. More than half of Austrian survey participants in HVC and LVC estimated the risk of secondary spleen rupture around $0-10 \%$, the other half around $10-30 \%$, which is in concordance with older studies reporting around $14 \%$ incidence [31]. Nevertheless, half of the survey participants overestimate the risk, leading to prolonged hospital stay or secondary splenectomy and failure of NOM [32].

Several prognostic factors for failure of NOM are reported in the literature [3, 4]. Based on this, relevant criteria for omission of ICU observation were analysed in our survey. Regressive haemoperitoneum (69\%), completely pain free $(73 \%)$ and increasing haemoglobin/haematocrit levels $(53 \%)$ were selected as most relevant. This is partially concordant with other analyses, where awareness is required in patients aged 40 years or older, with an International Severity Score (ISS) of $\geq 25$, or in those with injury grade $\geq$ III [3]. Other parameters that might be helpful in predicting the failure of NOM are lactate levels on admission, necessity of transfusion, crystalloid resuscitation and a drop in haematocrit levels during the first hour after admission [33].

In our survey, $93 \%$ of departments stated that the management of blunt hepatic and splenic trauma in Austria is not standardised and NOM is still based on individual necessities. However, most centres $(60 \%)$, independent of volume, agreed that a standardised algorithm for NOM would be helpful. Interestingly, one third of participants do not find standard algorithms useful, stating that NOM should remain individual for each patient. In fact, only around $40 \%$ follow defined protocols. These results are again in line with international trends. According to Peitzman et al., only one third of trauma centres have well-established NOM protocols for spleen injuries [34]. Only $20 \%$ of experts from the AAST consider that the protocol established in their institution is well supported by the available literature [25].

Multidisciplinary management is now conducted worldwide for the treatment of these patients. Single-centre studies have reported significant changes in diagnosis and treatment plans, if interdisciplinarity is integrated. Furthermore, regular audits allow for implementation of clinical practice guidelines and may help recruiting cases for clinical trials. However, $71 \%$ of Austrian departments, without significant differences between HVC and LVC, discuss cases only on a day-by-day basis, but $27 \%$ established routine audits. Studies from the United Kingdom showed that special training of multidisciplinary teams led to better team dynamics and communication, improved patient satisfaction and improved clinical outcome [35]. In suboptimal settings, such as small community hospitals with limited resources and rural areas, boundaries in diagnosis and management can be overcome or at least be improved with audits, especially by integrating the use of video-conferencing facilities [35].

The main limitation of this study, as inherent with all survey studies, is the incomplete response rate. Hence, no evidence-based conclusions can be drawn. Nevertheless, more than $60 \%$ of contacted departments responded to the survey $(n=125)$, which would be a reasonable rate, but only $36 \%(n=45)$ returned complete questionnaires. However, the average response rate to online surveys is approximately $30 \%$, therefore these figures are in line with the acceptable response range [11], and we are confident that the results represent a realistic picture of the national status. Survey research in healthcare is an important tool to collect information about healthcare delivery, service use and overall issues relating to quality of care. The survey was performed at trauma and surgical units to obtain a realistic picture of the current structure of blunt abdominal treatment in our country. Details of NOM may be influenced by the type of department in charge of decision making for abdominal trauma, a topic that has not been further evaluated in our survey. However, the differentiated analysis between HVC and LVC interestingly showed no relevant differences in the management of blunt abdominal injuries. In future evaluations, different treatment strategies in individual centres should furthermore be linked to outcome data such as morbidity, mortality and cost effectiveness. Regular national audits should be applied to ensure appropriate outcomes.

\section{Conclusions}

Besides the low evidence level created from a survey study, this multicentre questionnaire shows a signif- 
icant interhospital variability of hepatic and splenic NOM in Austria. The use of standard operating procedures to direct NOM of blunt hepatic and splenic injuries is uncommon in many centres, leading to controversial opinions in a number of questions on clinical practice, such as timing of mobilisation and thromboprophylaxis as well as length of intensified observation and post-discharge follow-up. Despite international efforts of increasing patient volume per centre to improve outcome, the number of LVC participating in our study clearly shows that the process of centralisation is still in progress in Austria-a country where the main ambition has always been to provide common access to high quality healthcare for the general population regardless of their socioeconomic status or geographic location. In this perspective, the present study could contribute to the creation of consensual protocols and clinical follow-up guidelines in Austria to assist in daily decision-making and optimising outcome in the long-term.

Funding This review paper was not sponsored by any organizations and we have no financial relationships.

Funding Open access funding provided by University of Innsbruck and Medical University of Innsbruck.

Conflict of interest M. Fodor, F. Primavesi, D. Morell-Hofert, M. Haselbacher, E. Braunwarth, B. Cardini, E. Gassner, D. Öfner and S. Stättner declare that they have no competing interests.

Open Access This article is distributed under the terms of the Creative Commons Attribution 4.0 International License (http://creativecommons.org/licenses/by/4.0/), which permits unrestricted use, distribution, and reproduction in any medium, provided you give appropriate credit to the original author(s) and the source, provide a link to the Creative Commons license, and indicate if changes were made.

\section{References}

1. Poole GV, Ward EF, Muakkassa FF, Hsu HS, Griswold JA, Rhodes RS. Pelvic fracture from major blunt trauma. Outcome is determined by associated injuries. Ann Surg. 1991;213(6):532-8. discussion 8-9.

2. Kornprat P, Uranues S, Salehi B, Hoebarth G, Buchinger W, Kuttnig M, et al. Preliminary results of a prospective study of nonoperative treatment of splenic injuries caused by blunt abdominal trauma. Eur Surg. 2007;39(1):33-8.

3. Olthof DC, Joosse P, van der Vlies CH, de Haan RJ, Goslings JC. Prognostic factors for failure of nonoperative management in adults with blunt splenic injury: a systematic review. J Trauma Acute Care Surg. 2013;74(2):546-57.

4. Raza M, Abbas Y, Devi V, Prasad KV, Rizk KN, Nair PP. Non operative management of abdominal trauma - a 10 years review. World J Emerg Surg. 2013;8:14.

5. Lucas CE, Ledgerwood AM. Changing times and the treatment ofliver injury. Am Surg. 2000;66(4):337-41.

6. Cogbill TH, Moore EE, Jurkovich GJ, Morris JA, Mucha P, Shackford SR, et al. Nonoperative management of blunt splenic trauma: a multicenter experience. J Trauma. 1989;29(10):1312-7.

7. Stassen NA, Bhullar I, Cheng JD, Crandall ML, Friese RS, Guillamondegui OD, et al. Selective nonoperative man- agement of blunt splenic injury: an Eastern Association for the Surgery of Trauma practice management guideline. JTrauma Acute Care Surg. 2012;73(5 Suppl4):294-300.

8. Stassen NA, Bhullar I, Cheng JD, Crandall M, Friese R, Guillamondegui $\mathrm{O}$, et al. Nonoperative management of blunt hepatic injury: an Eastern Association for the Surgery of Trauma practice management guideline. JTrauma Acute Care Surg. 2012;73(5 Suppl4):288-93.

9. Fodor M, Primavesi F, Morell-Hofert D, Haselbacher M, Braunwarth E, Cardini B, et al. Non-operative management of blunthepatic and splenic injuries-practical aspects and value of radiological scoring systems. European Surgery. Vienna: Springer; 2018.

10. Statistik Austria. https://www.statistik.at/web_de/ statistiken/menschen_und_gesellschaft/gesundheit/ unfaelle/strassenverkehrsunfaelle/index.html

11. Raptis DA, Clavien PA. CommitteeIH-P-BAIEaT.Evaluation of Hepato-Pancreato-Biliary (HPB) fellowships: an international survey of programme directors. HPB (Oxford). 2011;13(4):279-85.

12. Malhotra AK, Latifi R, Fabian TC, Ivatury RR, Dhage S, Bee TK, et al. Multiplicity of solid organ injury: influence on management and outcomes after blunt abdominal trauma. JTrauma. 2003;54(5):925-9.

13. Cirocchi R, Boselli C, Corsi A, Farinella E, Listorti C, Trastulli $\mathrm{S}$, et al. Is non-operative management safe and effective for all splenic blunt trauma? A systematic review. Crit Care. 2013;17(5):R185.

14. Coccolini F, Montori G, Catena F, KlugerY, BifflW, Moore EE, et al. Splenic trauma: WSES classification and guidelines for adult and pediatric patients. World J Emerg Surg. 2017;12:40.

15. Mirvis SE, Whitley NO, Vainwright JR, Gens DR. Blunt hepatic trauma in adults: CT-based classification and correlation with prognosis and treatment. Radiology. 1989;171(1):27-32.

16. Coccolini F, Montori G, Catena F, Di Saverio S, Biffl W, Moore EE, et al. Liver trauma: WSES position paper. World JEmerg Surg. 2015;10:39.

17. London JA, Parry L, Galante J, Battistella F. Safety of early mobilization of patients with blunt solid organ injuries. Arch Surg. 2008;143(10):972-6. discussion 7.

18. Von Rueden KT, Harris JR. Pulmonary dysfunction related to immobility in the trauma patient. AACN Clin Issues. 1995;6(2):212-28.

19. Lawson DE, Jacobson JA, Spizarny DL, Pranikoff T. Splenic trauma: value of follow-up CT. Radiology. 1995;194(1):97-100.

20. Juyia RF, Kerr HA. Return to play after liver and spleen trauma. Sports Health. 2014;6(3):239-45.

21. Lynch JM, Meza MP, Newman B, Gardner MJ, Albanese CT. Computed tomography grade of splenic injury is predictive of the time required for radiographic healing. JPediatr Surg. 1997;32(7):1093-5. discussion 5-6.

22. Clancy AA, Tiruta C, Ashman D, Ball CG, Kirkpatrick AW. The song remains the same although the instruments are changing: complications following selective non-operative management of bluntspleen trauma: a retrospective review of patients at a level I trauma centre from 1996 to 2007. JTrauma Manag Outcomes. 2012;6(1):4.

23. Navarro O, Babyn PS, Pearl RH. The value of routine followup imaging in pediatric blunt liver trauma. Pediatr Radiol. 2000;30(8):546-50.

24. Allins A, Ho T, Nguyen TH, Cohen M, Waxman K, Hiatt JR. Limited value of routine followup CT scans in nonoperative management of blunt liver and splenic injuries. Am Surg. 1996;62(11):883-6. 
25. Zarzaur BL, Kozar RA, Fabian TC, Coimbra R. A survey of American association for the surgery of trauma member practices in the management of blunt splenic injury. JTrauma. 2011;70(5):1026-31.

26. Rostas JW, Manley J, Gonzalez RP, Brevard SB, Ahmed N, Frotan MA, et al. The safety of low molecular-weight heparin after blunt liver and spleen injuries. Am J Surg. 2015;210(1):31-4.

27. Eberle BM, Schnüriger B, Inaba K, Cestero R, Kobayashi L, Barmparas G, et al. Thromboembolic prophylaxis with low-molecular-weight heparin in patients with blunt solid abdominal organ injuries undergoing nonoperative management: current practice and outcomes. J Trauma. 2011;70(1):141-6. discussion 7.

28. Joseph B, Pandit V, Harrison C, Lubin D, Kulvatunyou N, Zangbar B, et al. Early thromboembolic prophylaxis in patients with blunt solid abdominal organ injuries undergoing nonoperative management: is it safe? Am J Surg. 2015;209(1):194-8.

29. Fata P, Robinson L, Fakhry SM. A survey of EAST member practices in blunt splenic injury: a description of current trends and opportunities for improvement. J Trauma. 2005;59(4):836-41. discussion 41-2.

30. Crawford RS, Tabbara M, Sheridan R, Spaniolas K, Velmahos GC. Early discharge after nonoperative management for splenic injuries: increased patient risk caused by late failure? Surgery. 2007;142(3):337-42.

31. Olsen WR, Polley TZ. A second look at delayed splenic rupture. Arch Surg. 1977;112(4):422-5.

32. McIntyre LK, SchiffM, Jurkovich GJ. Failure of nonoperative management of splenic injuries: causes and consequences. Arch Surg. 2005;140(6):563-8. discussion 8-9.

33. Yanar H, Ertekin C, Taviloglu K, Kabay B, Bakkaloglu H, Guloglu R. Nonoperative treatment of multiple intra-abdominal solid organ injury after blunt abdominal trauma. JTrauma. 2008;64(4):943-8.

34. Peitzman AB, Heil B, Rivera L, Federle MB, Harbrecht BG, Clancy KD, et al. Blunt splenic injury in adults: Multiinstitutional Study of the Eastern Association for theSurgery of Trauma. JTrauma. 2000;49(2):177-87. discussion 87-9.

35. El Saghir NS, Keating NL, Carlson RW, Khoury KE, Fallowfield L. Tumor boards: optimizing the structure and improving efficiency of multidisciplinary management of patients with cancer worldwide. Am Soc Clin Oncol Educ Book. 2014;34:e461-e6.

Publisher's Note Springer Nature remains neutral with regard to jurisdictional claims in published maps and institutional affiliations. 